\title{
Benefits and adverse effects of hydroxychloroquine, methotrexate and colchicine: searching for repurposable drug candidates
}

\author{
Durga Prasanna Misra ${ }^{1}\left[\right.$ D $\cdot$ Armen Yuri Gasparyan $^{2}$ (D) $\cdot$ Olena Zimba $^{3}$ (i)
}

Received: 10 August 2020 / Accepted: 21 August 2020 / Published online: 2 September 2020

(c) Springer-Verlag GmbH Germany, part of Springer Nature 2020

\begin{abstract}
Repurposing of antirheumatic drugs has garnered global attention. The aim of this article is to overview available evidence on the use of widely used antirheumatic drugs hydroxychloroquine, methotrexate and colchicine for additional indications. Hydroxychloroquine has endothelial stabilizing and anti-thrombotic effects. Its use has been explored as an adjunctive therapy in refractory thrombosis in antiphospholipid syndrome. It may also prevent recurrent pregnancy losses in the absence of antiphospholipid antibodies. Hydroxychloroquine favourably modulates atherogenic lipid and glycaemic profiles. Methotrexate has been tried for modulation of cardiovascular events in non-rheumatic clinical conditions, although a large clinical trial failed to demonstrate a benefit. Colchicine has been shown to successfully reduce the risk of recurrent cardiovascular events in a large multicentric trial. Potential antifibrotic effects of colchicine require further exploration. Hydroxychloroquine, methotrexate and colchicine are also being tried at different stages of the ongoing Coronavirus Disease 19 (COVID-19) pandemic for prophylaxis and treatment. While the use of these agents is being diversified, their adverse effects should be timely diagnosed and prevented. Hydroxychloroquine can cause retinopathy and rarely cardiac and auditory toxicity, retinopathy being dose and time dependent. Methotrexate can cause transaminitis, cytopenias and renal failure, particularly in acute overdoses. Colchicine can rarely cause myopathies, cardiomyopathy, cytopenias and transaminitis. Strong evidence is warranted to keep balance between benefits of repurposing these old antirheumatic drugs and risk of their adverse effects.
\end{abstract}

Keywords Hydroxychloroquine $\cdot$ Methotrexate $\cdot$ Colchicine $\cdot$ Thrombosis $\cdot$ Cardiovascular risk $\cdot$ Fibrosis

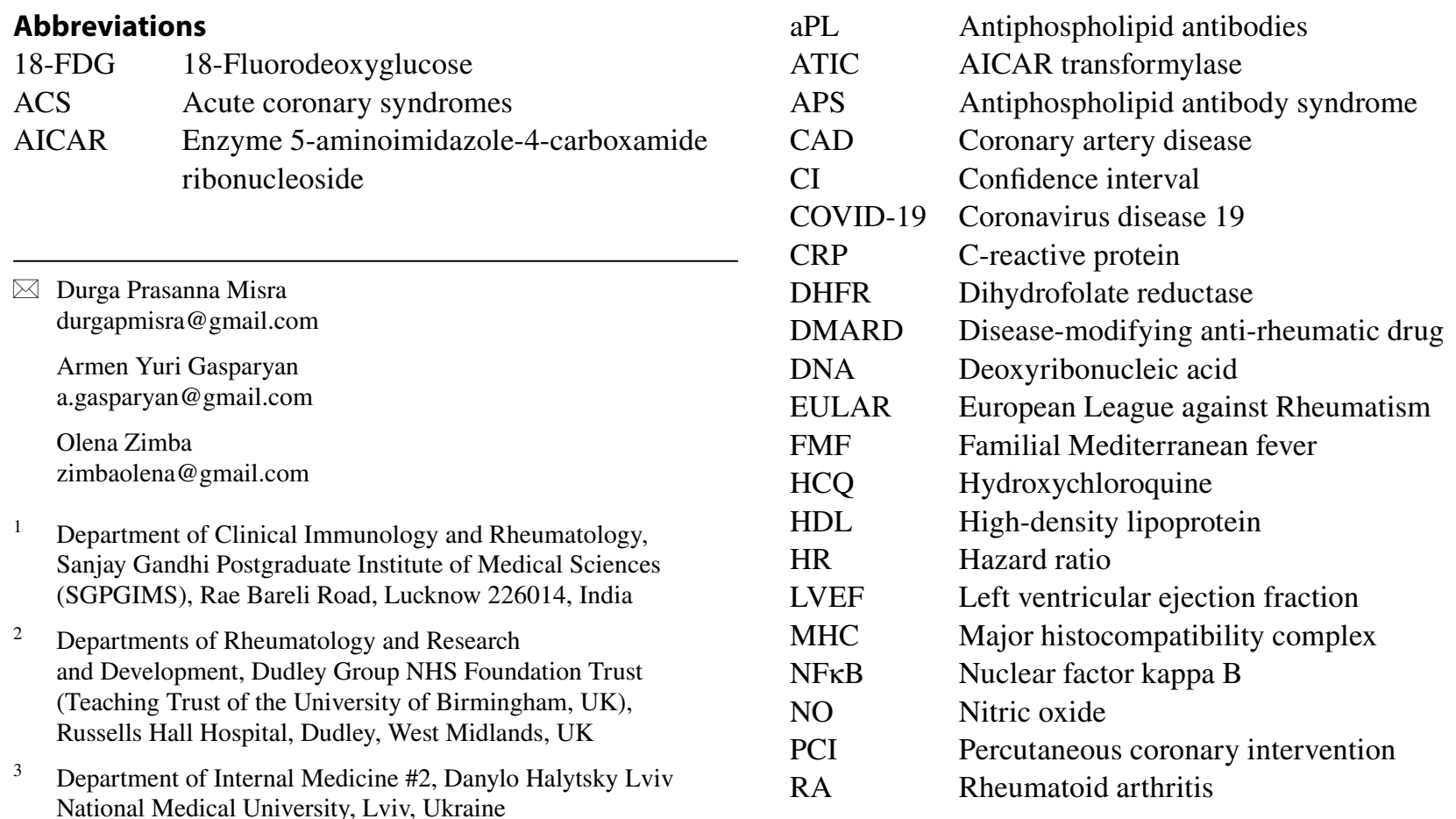




$\begin{array}{ll}\text { RCT } & \text { Randomized controlled trial } \\ \text { RNA } & \text { Ribonucleic acid } \\ \text { SLE } & \text { Systemic lupus erythematosus } \\ \text { STEMI } & \text { ST segment elevation myocardial infarction } \\ \text { UDCA } & \text { Ursodeoxycholic acid }\end{array}$

\section{Introduction}

Rheumatologists are at the forefront of research and practice of using various anti-inflammatory and immunomodulatory drugs. Their decades-long clinical experience can be valuable at the time of drug repurposing and attempting to use widely tested antirheumatic drugs for newer indications [1]. Numerous immunomodulatory drugs have been employed to induce remission of autoimmune disease and prevent adverse effects of concomitant high-dose corticosteroid and other synthetic disease-modifying anti-rheumatic drug (DMARD) therapies, particularly in elderly patients and those with comorbidities [2]. Although precise mechanisms of action of most old antirheumatic drugs remain unknown, these are exemplified for their relative safety and utility, allowing corticosteroid dose tapering and long-term disease control.

A Scopus search conducted on 10th August 2020 of the number of publications mentioning these drugs to this day revealed an ongoing interest in these drugs in the published literature. Overall, 163,999 documents are retrievable for methotrexate, 38,713 for colchicine, and 22,852 for hydroxychloroquine (Fig. 1).

The repurposing of traditionally used DMARDs has garnered significant attention recently with the advent of the
Coronavirus disease 19 (COVID-19) pandemic [3]. This opinion article aims to sensitize readers towards the potential utility of these drugs in cardiovascular disease and fibrosis (both of which have been reported as sequelae of COVID19) [4-6] as well as serve as a reminder of their adverse effect profile. Such potential adverse effects need to be kept in mind by specialists who do not routinely use these drugs when they are explored for other indications. In this article we overview some of the effects of hydroxychloroquine, methotrexate and colchicine and reflect on their positive and negative sides to guide repurposing of drug therapies. The choice of articles for review reflects the authors' understanding and viewpoints regarding the potential therapeutic roles of these drugs.

\section{Hydroxychloroquine}

Hydroxychloroquine (HCQ), a relatively safe derivative of chloroquine, is effectively used for the treatment of systemic lupus erythematosus and other inflammatory rheumatic diseases. It acts by accumulating in lysosomes, where its basic $\mathrm{pH}$ modifies the normally acidic milieu. This interferes with the loading and presentation of antigens by class II major histocompatibility complex (MHC) protein. Also, it interferes with the activation of toll-like receptors (TLR) by deoxyribonucleic acid (DNA) and ribonucleic acid (RNA) moieties [7].

The use of HCQ reduces the risk of thrombosis and diminishes vascular inflammation and endothelial dysfunction in a murine model of antiphospholipid syndrome (APS) [8]. In 22 patients with antiphospholipid antibodies (aPL)
Fig. 1 Annual publication activity in Scopus as of August 10, 2020 with the keywords "methotrexate", "colchicine" and "hydroxychloroquine" for articles published up to 2020 . Graph might appear to dip for some entries as the year 2020 is still ongoing. The graph seems to suggest that there is still ongoing interest in these older disease-modifying antirheumatic drugs

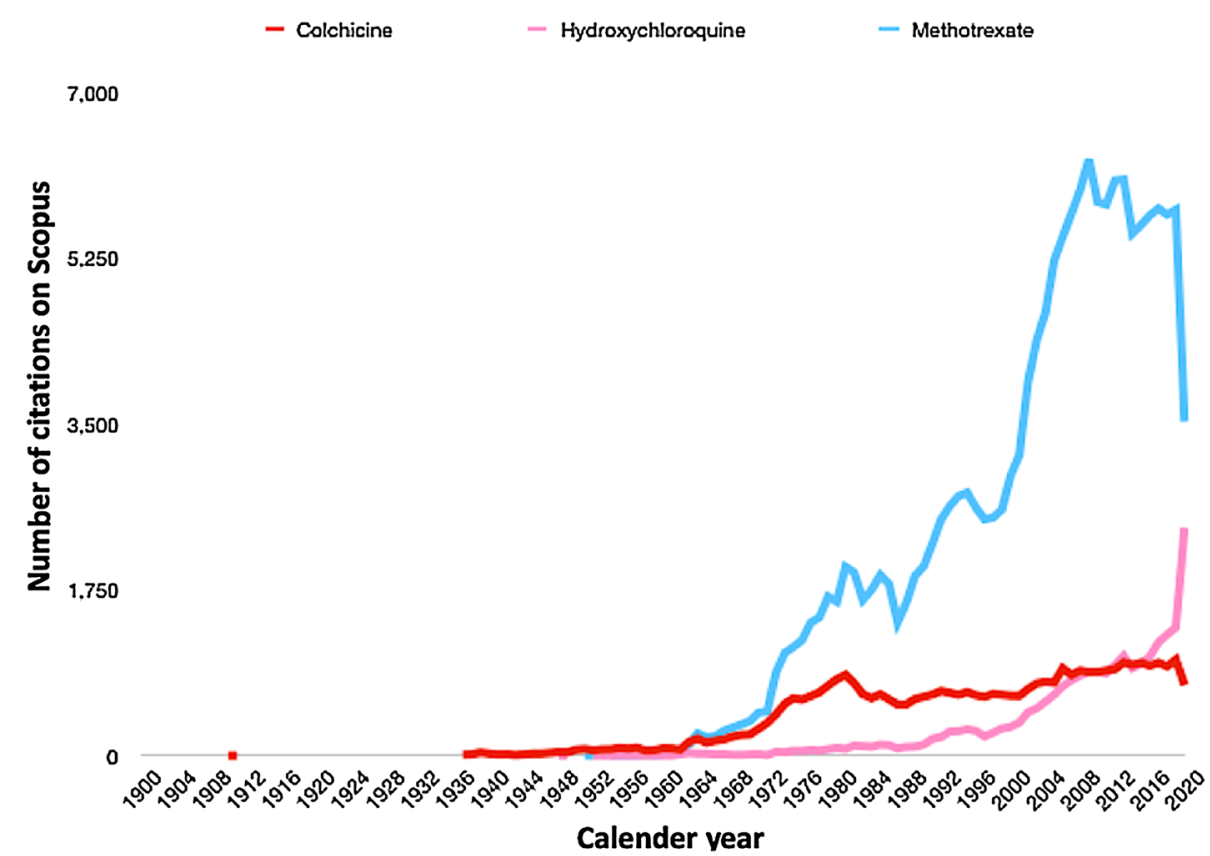


treated with HCQ, levels of soluble tissue factor reduced after three months of HCQ therapy. However, other markers of thrombogenic potential, such as annexin 5 activity and anti-domain 1 immunoglobulin G activity, and complement activation did not differ [9]. In 189 patients with systemic lupus erythematosus (SLE) from Italy (43 positive for aPL) followed up over a median of 13 years, there was a markedly reduced risk of vascular events in those treated with HCQ for five years (hazard ratio [HR] 0.04, 95\% confidence interval [CI] 0.004-0.48) [10]. Another retrospective study analysed insurance databases in Taiwan (1946 patients with SLE on HCQ during first year compared with a group of SLE patients not on HCQ during the same period) [11]. Over a mean period of 7.4 years, HCQ therapy was associated with a small but non-significant reduction in the risk of vascular events (HR 0.91, 95\% CI 0.71-1.15). The data was limited due to the lack of data about aPL for these patients [11].

A multicentric randomized controlled trial (RCT) reported 20 patients with aPL positivity in the absence of any systemic autoimmune disease who received either HCQ or placebo. Over a mean of 1.7 years of follow-up, neither group developed thrombotic events, resulting in premature termination of the trial and a subsequent inability to draw meaningful conclusions [12]. Another recent open-label study in Greece with primary APS treated with standard anticoagulation regimen (with or without antiplatelets) randomized 50 patients to receive either HCQ in addition to standard care or standard care only. Over mean 2.6 years follow-up, those on HCQ had a lower risk of incident thrombosis, which did not remain significant (HR 0.09, 95\% CI 0.01-1.26) after adjusting for confounders. Possibly, a larger sample size might have attained results with statistical significance [13]. The European League against Rheumatism (EULAR) recommends HCQ therapy (based on expert opinion and anecdotal evidence) for patients with APS and recurrent pregnancy loss despite optimal doses of anticoagulation and antiplatelet agents during pregnancy [14].
Various ongoing clinical trials are evaluating the role of add-on HCQ in APS. The HIBISCUS multicentre multinational trial is evaluating the effect of HCQ (400 mg daily for the duration of pregnancy) compared to placebo, in addition to standard therapy (i.e. preventative dose of low molecular weight heparin with aspirin), on live births in women with primary APS. A related study, the HIBISCUS-T trial, is evaluating the preventative role for recurrent thrombosis in patients with thrombotic APS treated with HCQ or placebo in addition to oral anticoagulation with vitamin $\mathrm{K}$ antagonists for 24 months [15]. The HYPATIA trial is another multicentric trial spanning multiple European nations assessing the effect of HCQ in a placebo-controlled RCT involving patients with either APS or persistent aPL positivity. This trial intends to assess the impact of HCQ, started before pregnancy and continued for 9 months, on pregnancy morbidity associated with aPL, i.e. a composite of early pregnancy losses, pre-term delivery ( $<34$ weeks) or placental insufficiency [16]. Another French multicentric trial, the HYDROSAPL trial, is also assessing the effect of HCQ on pregnancy outcomes in primary APS [17]. Apart from APS, HCQ is also being explored for its potential utility in patients with recurrent pregnancy loss. In this French RCT (the BBQ trial), patients with recurrent pregnancy loss shall be treated with HCQ $400 \mathrm{mg}$ daily or placebo starting pre-conception until the $10^{\text {th }}$ week of pregnancy, with the objective of assessing whether treatment improves pregnancy outcomes in such patients [18].

Other cardioprotective effects of HCQ, apart from reduction of thrombotic events, have also been proposed. In a model of induced myocardial infarction in rats, the administration of HCQ was associated with reduced apoptosis in the infarcted myocardial tissue after 12 weeks [19]. In patients with rheumatoid arthritis (RA) and Sjogren's syndrome, the use of HCQ has been associated with a reduced risk of developing diabetes mellitus (Table 1) [20-23]. In animal models of insulin resistance induced by a high fat

Table 1 Hydroxychloroquine and the risk of incident diabetes mellitus in cohort studies of patients with rheumatic diseases

\begin{tabular}{|c|c|c|c|}
\hline References & Country/database & Number of individuals & Salient findings \\
\hline 0$] / 2017$ & $\begin{array}{l}\text { USA/National Data Bank } \\
\text { for Rheumatic Diseases }\end{array}$ & 13,6 & $\begin{array}{l}\text { Over a median of } 4.6 \text { years of follow-up, the use of HCQ reduced the risk of } \\
\text { developing DM (HR } 0.67,95 \% \text { CI } 0.57-0.8)\end{array}$ \\
\hline$[21] / 2017$ & Taiwan/insurance database & $\begin{array}{l}\text { 84,989 patients with } \\
\text { AS, RA, PS, PsA }\end{array}$ & $\begin{array}{l}\text { Use of HCQ reduced risk of DM (HR } 0.7,95 \% \text { CI } 0.63-0.78) \text {. Use of HCQ } \\
\text { along with anti-TNF agents also reduced risk of DM (HR 0.49, 95\% CI } \\
0.36-0.66)\end{array}$ \\
\hline$[22] / 2019$ & USA/Corrona dataset & 21,775 patients $v$ & $\begin{array}{l}\text { Use of HCQ reduced the risk of diabetes non-significantly compared to other } \\
\text { non-biological DMARDs (adjusted HR based on a propensity score model } \\
0.45,95 \% \text { CI } 0.13-1.53 \text { ) }\end{array}$ \\
\hline$[23] / 2019$ & Taiwan/insurance database & 7774 patients with SS & Use of HCQ decreased risk of new-onset DM (HR 0.51, 95\% CI 0.28-0.96) \\
\hline
\end{tabular}

$A S$ ankylosing spondylitis, $C I$ confidence intervals, $D M$ diabetes mellitus, DMARD disease-modifying antirheumatic drugs, $H C Q$ hydroxychloroquine, $H R$ hazard ratio, $R A$ rheumatoid arthritis, $P S$ psoriasis, $P S A$ psoriatic arthritis, $S S$ Sjogren's syndrome, TNF tumour necrosis factor alpha, USA United States of America 
diet, the addition of HCQ was associated with better survival of the insulin-producing islet of Langerhans, as well as decreased release of inflammatory adipokines and markers of endothelial stress. Thus, a beneficial effect of HCQ on mechanisms driving insulin resistance has been postulated [24]. In a small proof-of-concept study of patients with type 2 diabetes mellitus $(n=17)$ inadequately controlled with a combination of metformin and sulfonylurea, the addition of HCQ $400 \mathrm{mg}$ daily was compared with pioglitazone $45 \mathrm{mg}$ daily. Although pioglitazone was more effective in reducing levels of glycated haemoglobin and achieving glycaemic control, nearly two-thirds of the patients on HCQ could attain less than $7.5 \%$ level of glycated haemoglobin [25]. Another small clinical trial of 39 patients with pre-diabetes to receive HCQ $(n=20)$ or placebo $(n=19)$ demonstrated improvements in glycaemic control over 4 months HCQ therapy [26]. These studies suggest the potential for HCQ as an add-on agent for diabetes mellitus. However, until the utility of HCQ is proven for the indications of diabetes mellitus, dyslipidemia or cardiovascular disease, its indiscriminate use for these indications should be avoided [27]. Considering the evidences for anti-platelet and anti-thrombotic actions of HCQ, as well as favourable lipid profile in patients treated with HCQ, it is possible that this drug might have an adjuvant role in the prevention of incident or recurrent cardiovascular events. This might need exploration in future clinical trials [28].

\section{Methotrexate}

Methotrexate is the first-line DMARD for the management of inflammatory arthritides such as rheumatoid arthritis and psoriatic arthritis. The drug's anti-folate mechanism of action is historically implicated in the treatment of neoplastic (lymphoblastic) diseases. The immunosuppressive action of low-dose methotrexate therapy (up to $25 \mathrm{mg} /$ week) primarily relies on the inhibition of the enzyme 5-aminoimidazole-4-carboxamide ribonucleoside (AICAR) transformylase (ATIC), resulting in higher levels of AICAR that inhibits adenosine monophosphate deaminase and adenosine deaminase. The latter results in higher extracellular levels of adenine, further converted to adenosine, which acts via adenosine receptors to exert antiinflammatory actions. Further, inhibition of the enzyme dihydrofolate reductase (DHFR) also results in uncoupling of nitric oxide (NO) synthetase, by increasing levels of dihydrobiopterin and tetrahydrobiopterin, resulting in greater release of $\mathrm{NO}$ as opposed to reactive oxygen species. Methotrexate also exerts an effect to decrease downstream inflammatory signalling operating via nuclear factor kappa $\mathrm{B}(\mathrm{NFkB})$. While supplementation with folic acid antagonizes the anti-folate action of methotrexate (responsible for most of its side effects), it does little to inhibit other anti-inflammatory pathways [29].

Recently accumulated evidence suggests a favourable modulation of cardiovascular risk in patients with rheumatic diseases treated with methotrexate $[30,31]$. A pilot study evaluated the safety of methotrexate therapy ( $5 \mathrm{mg} /$ week) in 16 patients with coronary artery disease undergoing percutaneous coronary intervention (PCI), started 2 weeks prior to the procedure and continued for 8 weeks after the procedure. No major safety signals were observed during this period [32]. A RCT, the TETHYS trial, evaluated the role of methotrexate in patients with ST segment elevation myocardial infarction (STEMI). Methotrexate administered at a dose of $0.05 \mathrm{mg} /$ $\mathrm{kg}$, repeated $6 \mathrm{~h}$ after $\mathrm{PCI}$ in 41 patients, was compared to 43 patients receiving placebo. This trial aimed to analyse area under the curve for serial measurements of creatine kinase and troponin I over the first $72 \mathrm{~h}$ after STEMI as a surrogate for area of infarct. No significant differences in the area of infarct measured either using creatine kinase or troponin I could be demonstrated between the two groups. A matter of concern was that the left ventricular ejection fraction (LVEF) was significantly lesser in patients treated with methotrexate compared to those receiving placebo, three months after completion of the treatment [33]. Another large multicentric RCT, the CIRT trial, evaluated the role of methotrexate in the secondary prevention of cardiovascular events. In this trial, patients who had a prior myocardial infarction or had triple vessel coronary artery disease on a background of diabetes mellitus or metabolic syndrome were randomized to receive methotrexate $15-20 \mathrm{mg} /$ week (2391 patients) or placebo (2395 patients). The primary endpoint was a composite of first occurrence of a major adverse cardiovascular event (non-fatal myocardial infarction, stroke, or death due to cardiovascular cause), later modified to include hospitalization for unstable angina resulting in a revascularization procedure. The results of this trial did not favour the use of methotrexate, with a small but insignificant reduction in the risk of developing the primary end point with methotrexate (HR 0.96, 95\% CI 0.79-1.16). Apart from a higher incidence of non-basal cell carcinoma in the group receiving methotrexate, no other significant safety signals were observed [34]. These studies do not seem to suggest a role for methotrexate in the prevention of cardiovascular events. An ongoing study (NCT03516903) is evaluating the targeting of left ventricular remodelling following STEMI with a nano-emulsion containing methotrexate. It remains to be seen whether such a strategy is useful to favourably modulate cardiovascular events. 


\section{Colchicine}

Colchicine has been used for the management of gout, familial Mediterranean fever (FMF) and Behcet's disease for decades [35]. It acts predominantly on neutrophils, monocytes and macrophages, decreasing their chemotaxis and release of various inflammatory cytokines. It interferes with the activation of the NLRP-3 inflammasome complex, thereby reducing the secretion of downstream cytokines such as IL-1ß and IL-18 [36, 37]. Knocking out the expression of NLRP-3 has been shown to reduce atherosclerosis as well as dampen the severity of myocardial injury following myocardial infarction in animal models [37]. In a murine model, the administration of colchicine improved survival and residual left ventricular function following myocardial infarction [38]. In a model of atherosclerosis induced by high fat diet in rabbits, the administration of colchicine reduced the uptake of 18 -fluodeoxyglucose (18FDG) in atherosclerotic plaques in the abdominal aorta. Moreover, in those rabbits with an increased cholesterol level in the peripheral blood, the burden of atherosclerotic plaques was significantly lesser along with greater circulating high-density lipoprotein (HDL) cholesterol [39]. In platelet-rich plasma from healthy volunteers, in-vitro treatment with colchicine significantly reduced platelet aggregation [40]. Refractoriness to colchicine when used in the longer term in disease states such as FMF has been associated with oxidative stress [41].

Several clinical studies have also assessed the modulation of cardiovascular risk by colchicine. In a parallelgroup observational study, 40 patients with coronary artery disease $(\mathrm{CAD})$ were treated with standard care and compared with another 40 patients also treated with colchicine $0.5 \mathrm{mg}$ daily in addition to standard care. Multivariable analysis revealed a significant effect of colchicine on the reduction of the volume of atherosclerotic plaques [42]. In a double-blind placebo-controlled trial of patients with CAD short-term colchicine therapy significantly reduced systemic inflammation and improved endothelial function [43]. In another study, patients with acute coronary syndromes (ACS) planned for PCI were randomized to treatment with add-on colchicine $(n=12)$ or just standard treatment $(n=13)$; those on colchicine had lower levels of chemokines, suggesting a reduction in local inflammatory activity at the site of pathology [44]. In the low dose colchicine for myocardial infarction (LoDoCo-MI) study, 237 patients with acute myocardial infarction were randomized to receive colchicine or placebo in addition to standard care, and C-reactive protein (CRP) levels were compared at 1 month. There was no significant difference in the proportions of patients attaining CRP levels lesser than $2 \mathrm{mg} / \mathrm{L}$ amongst patients treated with colchicine or placebo [45]. The ongoing LoDoCo2 trial is evaluating clinical cardiovascular outcomes in 5,552 patients with stable CAD treated with add-on colchicine or placebo [46]. The COLIN trial, evaluated treatment with add-on colchicine $(n=23)$ compared to placebo $(n=21)$ in patients with STEMI. Neither the primary outcome of difference in peak serum CRP during hospital admission between the two groups, nor secondary outcomes for differences in other biochemical, clinical or imaging outcomes (assessed by echocardiography or magnetic resonance imaging) at 1 month were successfully met [47]. A large multicentric trial evaluated the role of add-on colchicine $(n=2366)$ compared to placebo $(n=2379)$ in patients following myocardial infarction. The trial assessed the composite end-point of cardiovascular death, successful resuscitation after cardiac arrest, stroke, recurrent myocardial infarction, or angina requiring an intervention for revascularization. Followed up for a median 22.6 months, treatment with colchicine was associated with a reduced risk of the composite outcome compared to placebo (HR 0.77, 95\% CI 0.61-0.96). Gastrointestinal side-effects were similar, however, a greater incidence of pneumonia was observed in patients receiving colchicine ( $0.9 \%$ vs $0.4 \%$ on placebo) [48]. A recent systematic review also confirmed an atheroprotective effect of colchicine, with a significant reduction in future cerebrovascular ischemic events with colchicine (odds ratio $0.33,95 \%$ CI $0.15-0.70$ ) as observed in six clinical trials [49].

Colchicine may also exert anti-fibrotic effects. Clinical trials have demonstrated the potential role of colchicine in reducing the occurrence of atrial fibrillation following catheter ablation and cardiac surgeries [50]. It has been postulated that the modulation of atrial fibrosis by colchicine is mediated by its effects on interleukin-17 and renin-related pathways [51]. In a trial of patients undergoing coronary artery bypass grafting, perioperative treatment with colchicine was associated with reduction in post-surgical constrictive pericarditis [52]. In a rat model of hypertension induced by nephrectomy, treatment with colchicine ameliorated both glomerular and interstitial fibrosis in the remaining kidney [53]. In another animal model of renal injury induced by ureteric obstruction in rats, treatment with colchicine reduced both cortical fibrosis and tubulointerstitial injury [54]. Anecdotal reports also exist of the regression of retroperitoneal fibrosis in patients treated with colchicine [55]. In ten patients with keloids of the ear, treatment with colchicine for a month prior to keloid excision (along with pressure therapy and intralesional corticosteroid therapy in some) was associated with lack of recurrence of the keloids, although long-term follow-up data were unavailable for a majority of patients [56]. A potential role of colchicine in retarding the progression of oral submucous fibrosis has also been proposed [57]. Treatment with topical colchicine 
was also effective in reducing induced spinal epidural fibrosis in an experimental model in rats [58]. In a clinical trial involving patients with liver cirrhosis of different aetiologies, 37 patients were treated with standard treatment alone and another 37 with add-on colchicine. Followed up over a mean of 4.4 years, those treated with colchicine had a greater proportion of survival. Biochemical tests revealed that colchicine therapy was associated with lower serum levels of pro-collagen III peptide, a marker of fibrosis [59]. Another report described the combination of colchicine with ursodeoxycholic acid (UDCA) in patients with primary biliary cirrhosis who did not respond to UDCA alone. After a followup of 20 years, two-thirds of 18 patients continued to survive when treated with this combination treatment regimen [60]. It is possible that the anti-fibrotic effects of colchicine are mediated by its inhibitory actions on cytotoxic $\mathrm{T}$ lymphocytes as well as stimulation of endogenous mechanisms to regress fibrosis such as collagenases [61]. The potential antifibrotic effects of colchicine merit systematic evaluation by clinical trials in patients with systemic fibrosing diseases such as systemic sclerosis, or major organ fibrosis such as interstitial lung diseases.

\section{Exploration of repurposing of hydroxychloroquine, methotrexate and colchicine for coronavirus disease 19 (COVID-19)}

The COVID-19 pandemic has swept the world. Based on preliminary evidence, abnormal activation of the immune system and cytokine storm appear to be associated with disease severity. In this context, commonly used antirheumatic drugs are being explored in COVID-19 [3]. While hydroxychloroquine has been recommended for prophylaxis of high-risk contacts of COVID-19, there is little evidence at present to support this [62]. Occasional reports have even described patients on long term hydroxychloroquine therapy for rheumatic diseases who eventually developed COVID19 [63]. Overall, the level of enthusiasm regarding the use of hydroxychloroquine in COVID-19 has dampened due to mixed, often negative preliminary results of trials [64]. It has been hypothesized that the timing (early initiation) of hydroxychloroquine might play a role in protection against severe COVID-19, and the results of trials on chemoprophylaxis of COVID-19 with hydroxychloroquine might help answer this question [65]. In this context, it is necessary to mention that hydroxychloroquine (and its analogue, chloroquine) have been previously tried in other viral infections such as Zika virus and Ebola virus infections. While in-vitro efficacy had been observed, this did not translate into clinical efficacy, possibly due to the fact that the drugs were unable to attain a concentration in the endosome necessary for their anti-viral properties to take effect $[66,67]$. Anecdotal reports have suggested reduced severity of COVID-19 in patients on colchicine for other indications [68]. Another retrospective review of records of patients from an European centre compared 140 patients treated with standard of care (varying combinations of hydroxychloroquine, lopinavir/ritonavir and corticosteroids) with 122 patients treated with colchicine at $0.5-1 \mathrm{mg} / \mathrm{day}$ in addition to standard of care. Treatment with colchicine was associated with $85 \%$ reduction in hazard of death (hazard ratio $0.15,95 \%$ confidence intervals 0.06-0.37) [69]. However, in another recent study, six out of seven children with paediatric autoinflammatory syndromes who developed COVID-19 were on colchicine [70]. Ongoing clinical trials identified on a search on clinicaltrials.gov on 19 August 2020 are summarized in Table 2. The results of these trials shall help better understand the role of antirheumatic drugs in COVID-19 in the coming times.

It is increasingly being understood that recovery from COVID-19 is prolonged. Nearly one-half of patients with

Table 2 Ongoing clinical trials registered on clinicaltrials.gov exploring hydroxychloroquine, methotrexate and colchicine for coronavirus disease 19

\begin{tabular}{lll}
\hline Drug & $\begin{array}{l}\text { Number of trials regis- } \\
\text { tered as of 19th August, } \\
2020\end{array}$ & Aspects being explored \\
\hline Hydroxychloroquine & 229 & $\begin{array}{l}\text { Use of this drug alone or in combination of other agents, or compared to other agents (eg. } \\
\text { lopinavir/ritonavir, oseltamivir, favipiravir) for treatment of mild or severe COVID-19 } \\
\text { Chemoprophylaxis in contacts, health care workers }\end{array}$ \\
Methotrexate & 3 & $\begin{array}{l}\text { Reduction of progression to severe disease/ prevention of hospitalization in milder COVID-19 } \\
\text { Treatment of severe COVID-19 with methotrexate-containing nanoparticles } \\
\text { Risk of COVID-19 in patients with rheumatic diseases on drugs like methotrexate } \\
\text { Treatment of mild or severe COVID-19 } \\
\text { Prevention of progression of mild COVID-19 to severe disease } \\
\text { Treatment of myocardial injury in the context of COVID-19 }\end{array}$ \\
\hline
\end{tabular}

COVID-19 coronavirus disease 19 
COVID-19 from Italy had persistence of symptoms, including fatigue and joint pains, after 2 months of onset of clinical disease [5]. Neuro-cognitive disorders are also being described in COVID-19 survivors, at least in part driven by the endothelial injury due to the infection [71]. A significant proportion of patients with pneumonitis due to COVID-19 are likely to develop residual pulmonary fibrosis $[6,72]$. COVID-19 causes endothelial injury, and this might be responsible for long-term cardiovascular consequences of the disease, including a predisposition towards future cardiovascular events $[4,73]$. Therefore, age-old rheumatic drugs should be explored in such select recovered populations. Particularly, it can be hypothesized that the anti-fibrotic effects of colchicine might help in post-COVID-19 fibrosis, including lung fibrosis.

\section{Adverse effects of hydroxychloroquine, methotrexate and colchicine}

Table 3 summarizes side effects of the examined drugs. HCQ entails the risk of toxicities in the long term [74]. Ocular toxicity occurs in the form of retinal damage. Up to 1 in 20 patients might develop retinopathy after five years of using hydroxychloroquine, and this is often silent in the earlier stages [75]. In the later stages, macular vision may be affected, resulting in maculopathy and vision loss. Fundoscopy and perimetry are insensitive for picking up earlier changes of HCQ retinopathy. Recent recommendations

Table 3 Adverse effect profile

\begin{tabular}{ll}
\hline Drug & Major adverse effects \\
\hline Hydroxychloroquine & Retinopathy \\
& Cutaneous pigmentation \\
& Myopathy \\
& Cardiomyopathy \\
& Cardiac conduction defects \\
& Transaminitis \\
Methotrexate & Cytopenias (leuco- \\
& penia, neutropenia, \\
& thrombocytopenia) \\
& Acute kidney injury \\
& Mucositis \\
Colchicine & Diarrhoea \\
& Myopathy \\
& Neuropathy \\
\hline
\end{tabular}

${ }^{a}$ Risk of retinopathy generally occurs after years of use, and is related to cumulative doses. Those with renal failure or on concomitant tamoxifen therapy are at greater risk

${ }^{b}$ Per se, cardiac toxicity is very rare when hydroxychloroquine is used in the context of rheumatic diseases

${ }^{\mathrm{c}}$ Generally seen with methotrexate toxicity, such as in overdose (daily rather than weekly administration), or with concomitant underlying renal impairment suggest limiting the dose of HCQ to $5 \mathrm{mg} / \mathrm{kg} / \mathrm{day}$, since higher doses increase the risk of retinopathy after 5 years [76]. Before initiation of HCQ, baseline fundus evaluation with the macula assessment is recommended. If this is abnormal, more sensitive techniques such as spectral domain optical coherence tomography may be employed and repeated annually [76]. Other adverse effects HCQ-related skin pigmentation due to sun exposure, myopathy, and conduction blocks [77]. Cardiac toxicity may be potentiated by other drugs resulting in prolongation of the QT interval such as azithromycin [78]. Rare instances of sensorineural hearing loss have also been noted [79].

In the context of the ongoing COVID-19 pandemic, potential cardiac toxicity of HCQ has received widespread attention. However, speaking from personal experience as well as the published literature in rheumatic diseases [80], cardiac toxicity with HCQ (including QT prolongation) is very rare. Pharmacological interaction with azithromycin can potentially prolong QT interval when these two drugs are taken together, as has been tried in the management of COVID-19. Hence, due caution and pre-treatment with follow-up ECGs to seek any developing cardiac arrhythmias might be suggested if and when HCQ is combined with azithromycin or any other drug that can cause QT prolongation [81].

The adverse effect profile of methotrexate is mainly due to its antifolate actions [29]. Methotrexate toxicity can result in mucositis with oral ulcers, crusting of lips and diarrhoea, skin rashes, pancytopenia, transaminitis, and acute kidney injury [82]. The risk of toxicities is greater in the presence of renal failure. In a series of 120 patients with rheumatoid arthritis with concomitant renal impairment treated with methotrexate, nearly $30 \%$ developed features of methotrexate toxicity such as leucopenia, transaminitis and renal failure. Interestingly, a protective effect towards methotrexate toxicity in the presence of renal failure was observed in those individuals also taking hydroxychloroquine [83]. Acute methotrexate toxicity requires discontinuation of the drug and administration of folinic acid. Varying dose regimens of folinic acid are used, although often $15 \mathrm{mg} 6$ hourly for 10 doses is administered. Supportive treatment in the form of granulocyte colony stimulating factor, blood component transfusions, treatment of coexistent infections and nutritional care are also essential. Untreated methotrexate toxicity is associated with significant risk of mortality [82, 84]. Rarely, methotrexate may result in acute interstitial pneumonitis [85].

Colchicine therapy can result in diarrhoea and other gastrointestinal adverse effects. Long-term colchicine therapy is rarely associated with myopathy, polyneuropathy, transaminitis, and rhabdomyolysis [86]. The risk of toxicity is increased in the presence of renal failure. Also, coadministration with drugs inhibiting the enzyme cytochrome 
P450 oxidase 3A4 (CYP 3A4, such as clarithromycin) or the drug efflux protein p-glycoprotein portends a greater risk of adverse effects [87]. Occasional reports exist regarding the use of intravenous colchicine for prolonged periods for difficult to treat conditions such as familial Mediterranean fever. Despite intravenous use for longer term, little toxicity was observed in most patients, other than gastrointestinal side effects $[88,89]$. Drug withdrawal is recommended if such toxicities develop [86, 90].

Rarely, these drugs can have cardiovascular side effects also, as holds true for most anti-rheumatic drugs: methotrexate has been associated with pericardial and myocardial injury, colchicine rarely causes myocardial injury, and HCQ therapy occasionally results in conduction system abnormalities [91]. Clinicians should be aware for the risk of cardiac adverse events when these drugs are considered for repurposing and combined therapies in different patient cohorts.

\section{Future perspectives}

Keeping in mind the diverse impacts on different body systems seen with these drugs, it is imperative to generate high-quality evidence for the use of hydroxychloroquine, methotrexate and colchicine for other indications. They are already being tried in patients with cardiovascular disease for secondary prevention. Clinical trials might also attempt to decipher the potential utility of hydroxychloroquine, methotrexate or colchicine for primary prevention of cardiovascular events in a high risk population, such as those with metabolic syndrome and in individuals with inflammatory arthritides which predispose to greater cardiovascular risk. Furthermore, the potential anti-fibrotic effects of colchicine need to be systematically studied in local fibrosing diseases (such as idiopathic interstitial lung diseases) or systemic fibrosing diseases (such as systemic sclerosis) by suitably powered clinical trials. Although recent promising developments in vaccines for COVID-19 hold promise for community level prevention, these are still preliminary and it is likely that millions of individuals shall be infected before such vaccines come into regular clinical use [92]. Therefore, the role of drugs with anti-fibrotic potential such as colchicine in preventing or managing long-term sequelae of COVID-19 should also be explored.

\section{Conclusion}

Repurposing of old antirheumatic drugs is being attempted for numerous indications today. HCQ, methotrexate and colchicine are primarily used for favourable modulation of cardiovascular risk. Clinicians must be aware of the propensity of these drugs to cause side effects, such as cytopenias with methotrexate, retinopathy with HCQ, and gastrointestinal adverse effects with colchicine. In view of the risk of adverse events, it is essential to carefully consider the risk-benefit ratio before prescribing these drugs for newer, unlicensed indications, taking particular care to identify high-risk populations such as those with underlying renal impairment and avoid drug interactions. The role of these drugs in COVID19 shall be clarified in ongoing trials. It may also require evaluation whether these drugs have a role in managing sequelae of COVID-19.

Author contributions (1) Conception, design, collection of data, analysis and interpretation of data-DPM, AYG, OZ. (2) Writing the original draft-DPM; Critical revision-AYG, OZ. (3) Final approval of the version to be published-DPM, AYG, OZ. (4) Agreement to be accountable for all aspects of the manuscript in ensuring that questions related to the accuracy or integrity of any part of the work are appropriately investigated and resolved-DPM, AYG, OZ.

Funding None.

\section{Compliance with ethical standards}

Conflict of interest Durga Prasanna Misra declares that he has no conflict of interest, including no relationship with pharmaceutical companies. Armen Yuri Gasparyan declares that he has no conflict of interest, including no relationship with pharmaceutical companies. Olena Zimba declares that she has no conflict of interest, including no relationship with pharmaceutical companies.

Ethics approval Not applicable.

Consent to participate Not applicable.

Consent for publication Not applicable.

Availability of data and material Not applicable.

\section{References}

1. Kingsmore KM, Grammer AC, Lipsky PE (2020) Drug repurposing to improve treatment of rheumatic autoimmune inflammatory diseases. Nat Rev Rheumatol 16:32-52. https://doi.org/10.1038/ s41584-019-0337-0

2. Kerrigan SA, McInnes IB (2020) Reflections on 'older' drugs: learning new lessons in rheumatology. Nat Rev Rheumatol 16:179-183. https://doi.org/10.1038/s41584-020-0375-7

3. Misra DP, Agarwal V, Gasparyan AY, Zimba O (2020) Rheumatologists' perspective on coronavirus disease 19 (COVID-19) and potential therapeutic targets. Clin Rheumatol 39:2055-2062. https ://doi.org/10.1007/s10067-020-05073-9

4. Suri JS, Puvvula A, Biswas M et al (2020) COVID-19 pathways for brain and heart injury in comorbidity patients: a role of medical imaging and artificial intelligence-based COVID severity classification: a review. Comput Biol Med 103960. https://doi. org/10.1016/j.compbiomed.2020.103960.

5. Carfì A, Bernabei R, Landi F, for the Gemelli Against CovidPost-Acute Care Study Group (2020) Persistent symptoms in 
patients after acute COVID-19. JAMA 324:603-605. https://doi. org/10.1001/jama.2020.12603

6. Fraser E (2020) Long term respiratory complications of covid-19. BMJ 370:m3001. https://doi.org/10.1136/bmj.m3001

7. Schrezenmeier E, Dorner T (2020) Mechanisms of action of hydroxychloroquine and chloroquine: implications for rheumatology. Nat Rev Rheumatol 16:155-166. https://doi.org/10.1038/ s41584-020-0372-x

8. Miranda S, Billoir P, Damian L et al (2019) Hydroxychloroquine reverses the prothrombotic state in a mouse model of antiphospholipid syndrome: Role of reduced inflammation and endothelial dysfunction. PLoS ONE 14:e0212614. https://doi.org/10.1371/ journal.pone. 0212614

9. Schreiber K, Breen K, Parmar K, Rand JH, Wu XX, Hunt BJ (2018) The effect of hydroxychloroquine on haemostasis, complement, inflammation and angiogenesis in patients with antiphospholipid antibodies. Rheumatology (Oxford) 57:120-124. https:// doi.org/10.1093/rheumatology/kex378

10. Fasano S, Pierro L, Pantano I, Iudici M, Valentini G (2017) Longterm hydroxychloroquine therapy and low-dose aspirin may have an additive effectiveness in the primary prevention of cardiovascular events in patients with systemic lupus erythematosus. $\mathrm{J}$ Rheumatol 44:1032-1038. https://doi.org/10.3899/jrheum.16135 1

11. Hsu CY, Lin YS, Su YJ et al (2017) Effect of long-term hydroxychloroquine on vascular events in patients with systemic lupus erythematosus: a database prospective cohort study. Rheumatology (Oxford) 56:2212-2221. https://doi.org/10.1093/rheumatolo gy/kex357

12. Erkan D, Unlu O, Sciascia S et al (2018) Hydroxychloroquine in the primary thrombosis prophylaxis of antiphospholipid antibody positive patients without systemic autoimmune disease. Lupus 27:399-406. https://doi.org/10.1177/0961203317724219

13. Kravvariti E, Koutsogianni A, Samoli E, Sfikakis PP, Tektonidou MG (2020) The effect of hydroxychloroquine on thrombosis prevention and antiphospholipid antibody levels in primary antiphospholipid syndrome: A pilot open label randomized prospective study. Autoimmun Rev 19:102491. https://doi.org/10.1016/j.autre v.2020.102491

14. Tektonidou MG, Andreoli L, Limper M et al (2019) EULAR recommendations for the management of antiphospholipid syndrome in adults. Ann Rheum Dis 78:1296-1304. https://doi.org/10.1136/ annrheumdis-2019-215213

15. Belizna C, Pregnolato F, Abad S et al (2018) HIBISCUS: Hydroxychloroquine for the secondary prevention of thrombotic and obstetrical events in primary antiphospholipid syndrome. Autoimmun Rev 17:1153-1168. https://doi.org/10.1016/j.autre v.2018.05.012

16. Schreiber K, Breen K, Cohen $\mathrm{H}$ et al (2017) HYdroxychloroquine to Improve Pregnancy Outcome in Women with AnTIphospholipid Antibodies (HYPATIA) Protocol: a multinational randomized controlled trial of hydroxychloroquine versus placebo in addition to standard treatment in pregnant women with antiphospholipid syndrome or antibodies. Semin Thromb Hemost 43:562571. https://doi.org/10.1055/s-0037-1603359

17. Mekinian A, Vicaut E, Cohen J, Bornes M, Kayem G, Fain O (2018) Hydroxychloroquine to obtain pregnancy without adverse obstetrical events in primary antiphospholipid syndrome: French phase II multicenter randomized trial, HYDROSAPL. Gynecol Obstet Fertil Senol 46:598-604. https://doi.org/10.1016/j. gofs.2018.06.008

18. Pasquier E, de Saint-Martin L, Marhic G et al (2019) Hydroxychloroquine for prevention of recurrent miscarriage: study protocol for a multicentre randomised placebo-controlled trial BBQ study. BMJ Open 9:e025649. https://doi.org/10.1136/bmjop en-2018-025649
19. Zhou J, Li G, Wang ZH, Wang LP, Dong PJ (2013) Effects of low-dose hydroxychloroquine on expression of phosphorylated Akt and p53 proteins and cardiomyocyte apoptosis in peri-infarct myocardium in rats. Exp Clin Cardiol 18:e95-98

20. Ozen G, Pedro S, Holmqvist ME, Avery M, Wolfe F, Michaud K (2017) Risk of diabetes mellitus associated with disease-modifying antirheumatic drugs and statins in rheumatoid arthritis. Ann Rheum Dis 76:848-854. https://doi.org/10.1136/annrheumdi s-2016-209954

21. Chen HH, Chen DY, Lin CC, Chen YM, Lai KL, Lin CH (2017) Association between use of disease-modifying antirheumatic drugs and diabetes in patients with ankylosing spondylitis, rheumatoid arthritis, or psoriasis/psoriatic arthritis: a nationwide, population-based cohort study of 84,989 patients. Ther Clin Risk Manag 13:583-592. https://doi.org/10.2147/tcrm.S130666

22. Lillegraven S, Greenberg JD, Reed GW et al (2019) Immunosuppressive treatment and the risk of diabetes in rheumatoid arthritis. PLoS ONE 14:e0210459. https://doi.org/10.1371/journ al.pone. 0210459

23. Chen TH, Lai TY, Wang YH, Chiou JY, Hung YM, Wei JC (2019) Hydroxychloroquine was associated with reduced risk of newonset diabetes mellitus in patients with Sjogren syndrome. QJM 112:757-762. https://doi.org/10.1093/qjmed/hcz112

24. Abdel-Hamid AAM, Firgany AEL (2016) Favorable outcomes of hydroxychloroquine in insulin resistance may be accomplished by adjustment of the endothelial dysfunction as well as the skewed balance of adipokines. Acta Histochem 118:560-573. https://doi. org/10.1016/j.acthis.2016.06.002

25. Hsia SH, Duran P, Lee ML, Davidson MB (2020) Randomized controlled trial comparing hydroxychloroquine with pioglitazone as third-line agents in type 2 diabetic patients failing metformin plus a sulfonylurea: a pilot study. J Diabetes 12:91-94. https://doi. org/10.1111/1753-0407.12989

26. Sheikhbahaie F, Amini M, Gharipour M, Aminoroaya A, Taheri N (2016) The effect of hydroxychloroquine on glucose control and insulin resistance in the prediabetes condition. Adv Biomed Res 5:145. https://doi.org/10.4103/2277-9175.187401

27. Luthra A, Misra A (2015) The marketing of unproven drugs for diabetes and dyslipidaemia in India. Lancet Diabetes Endocrinol 3:758-760. https://doi.org/10.1016/s2213-8587(15)00328-9

28. Sun L, Liu M, Li R et al (2016) Hydroxychloroquine, a promising choice for coronary artery disease? Med Hypotheses 93:5-7. https ://doi.org/10.1016/j.mehy.2016.04.045

29. Cronstein BN, Aune TM (2020) Methotrexate and its mechanisms of action in inflammatory arthritis. Nat Rev Rheumatol 16:145154. https://doi.org/10.1038/s41584-020-0373-9

30. Misra DP, Shenoy SN (2017) Cardiac involvement in primary systemic vasculitis and potential drug therapies to reduce cardiovascular risk. Rheumatol Int 37:151-167. https://doi.org/10.1007/ s00296-016-3435-1

31. Popkova TV, Novikova DS, Gasparyan AY, Nasonov EL (2015) Cardiovascular effects of methotrexate in rheumatoid arthritis revisited. Curr Med Chem 22:1903-1910. https://doi. org/10.2174/0929867322666150415122039

32. Gouveia V, Oliveira DC, Tenorio E, Brito N, Sarinho E (2016) Percutaneous coronary intervention: safety of methotrexate and its possible benefits on restenosis after bare-metal stent deployment. Cardiol Res 7:104-109. https://doi.org/10.14740/cr468w

33. Moreira DM, Lueneberg ME, da Silva RL, Fattah T, Gottschall CAM (2017) MethotrexaTE THerapy in ST-Segment Elevation MYocardial InfarctionS: a randomized double-blind, placebocontrolled trial (TETHYS Trial). J Cardiovasc Pharmacol Ther 22:538-545. https://doi.org/10.1177/1074248417699884

34. Ridker PM, Everett BM, Pradhan A et al (2019) Low-dose methotrexate for the prevention of atherosclerotic events. N Engl J Med 380:752-762. https://doi.org/10.1056/NEJMoa1809798 
35. Gasparyan AY, Ayvazyan L, Yessirkepov M, Kitas GD (2015) Colchicine as an anti-inflammatory and cardioprotective agent. Expert Opin Drug Metab Toxicol 11:1781-1794. https://doi. org/10.1517/17425255.2015.1076391

36. Bhat A, Naguwa SM, Cheema GS, Gershwin ME (2009) Colchicine revisited. Ann N Y Acad Sci 1173:766-773. https://doi.org/ 10.1111/j.1749-6632.2009.04674.x

37. Martinez GJ, Celermajer DS, Patel S (2018) The NLRP3 inflammasome and the emerging role of colchicine to inhibit atherosclerosis-associated inflammation. Atherosclerosis 269:262-271. https ://doi.org/10.1016/j.atherosclerosis.2017.12.027

38. Fujisue K, Sugamura K, Kurokawa H et al (2017) Colchicine improves survival, left ventricular remodeling, and chronic cardiac function after acute myocardial infarction. Circ J 81:11741182. https://doi.org/10.1253/circj.CJ-16-0949

39. Cecconi A, Vilchez-Tschischke JP, Mateo J et al (2020) Effects of colchicine on atherosclerotic plaque stabilization: a multimodality imaging study in an animal model. J Cardiovasc Transl Res. https ://doi.org/10.1007/s12265-020-09974-7

40. Cimmino G, Tarallo R, Conte S et al (2018) Colchicine reduces platelet aggregation by modulating cytoskeleton rearrangement via inhibition of cofilin and LIM domain kinase 1. Vascul Pharmacol 111:62-70. https://doi.org/10.1016/j.vph.2018.09.004

41. Omma A, Sandikci SC, Kücüksahin O, Alisik M, Erel O (2017) Can the thiol/disulfide imbalance be a predictor of colchicine resistance in familial mediterranean fever? J Korean Med Sci 32:1588-1594. https://doi.org/10.3346/jkms.2017.32.10.1588

42. Vaidya K, Arnott C, Martinez GJ et al (2018) Colchicine therapy and plaque stabilization in patients with acute coronary syndrome: a CT coronary angiography study. JACC Cardiovasc Imaging 11:305-316. https://doi.org/10.1016/j.jcmg.2017.08.013

43. Kajikawa M, Higashi Y, Tomiyama H et al (2019) Effect of shortterm colchicine treatment on endothelial function in patients with coronary artery disease. Int J Cardiol 281:35-39. https://doi. org/10.1016/j.ijcard.2019.01.054

44. Tucker B, Kurup R, Barraclough J et al (2019) Colchicine as a novel therapy for suppressing chemokine production in patients with an acute coronary syndrome: a pilot study. Clin Ther 41:2172-2181. https://doi.org/10.1016/j.clinthera.2019.07.015

45. Hennessy T, Soh L, Bowman M et al (2019) The Low Dose Colchicine after Myocardial Infarction (LoDoCo-MI) study: a pilot randomized placebo controlled trial of colchicine following acute myocardial infarction. Am Heart J 215:62-69. https://doi. org/10.1016/j.ahj.2019.06.003

46. Nidorf SM, Fiolet ATL, Eikelboom JW et al (2019) The effect of low-dose colchicine in patients with stable coronary artery disease: the LoDoCo2 trial rationale, design, and baseline characteristics. Am Heart J 218:46-56. https://doi.org/10.1016/j. ahj.2019.09.011

47. Akodad M, Lattuca B, Nagot $\mathrm{N}$ et al (2017) COLIN trial: value of colchicine in the treatment of patients with acute myocardial infarction and inflammatory response. Arch Cardiovasc Dis 110:395-402. https://doi.org/10.1016/j.acvd.2016.10.004

48. Tardif JC, Kouz S, Waters DD et al (2019) Efficacy and safety of low-dose colchicine after myocardial infarction. N Engl J Med 381:2497-2505. https://doi.org/10.1056/NEJMoa1912388

49. Masson W, Lobo M, Molinero G, Masson G, Lavalle-Cobo A (2020) Role of colchicine in stroke prevention: an updated meta-analysis. J Stroke Cerebrovasc Dis 104756. https://doi. org/10.1016/j.jstrokecerebrovasdis.2020.104756

50. Deftereos SG, Vrachatis DA, Angelidis C et al (2019) The role of colchicine in treating postoperative and post-catheter ablation atrial fibrillation. Clin Ther 41:21-29. https://doi.org/10.1016/j. clinthera.2018.08.008

51. Yue H, Liang W, Gu J et al (2019) Comparative transcriptome analysis to elucidate the therapeutic mechanism of colchicine against atrial fibrillation. Biomed Pharmacother 119:109422. https ://doi.org/10.1016/j.biopha.2019.109422

52. Shojaeifard M, Pakbaz M, Beheshti R et al (2020) The effect of colchicine on the echocardiographic constrictive physiology after coronary artery bypass graft surgery. Echocardiography 37:399403. https://doi.org/10.1111/echo.14605

53. Guan T, Gao B, Chen G et al (2013) Colchicine attenuates renal injury in a model of hypertensive chronic kidney disease. Am J Physiol Renal Physiol 305:F1466-1476. https://doi.org/10.1152/ ajprenal.00057.2013

54. Kim S, Jung ES, Lee J, Heo NJ, Na KY, Han JS (2018) Effects of colchicine on renal fibrosis and apoptosis in obstructed kidneys. Korean J Intern Med 33:568-576. https://doi.org/10.3904/ kjim.2016.131

55. de Socio G, Verrecchia E, Fonnesu C, Giovinale M, Gasbarrini GB, Manna R (2010) Effectiveness of colchicine therapy in 4 cases of retroperitoneal fibrosis associated with autoinflammatory diseases. J Rheumatol 37:1971-1972. https://doi.org/10.3899/ jrheum. 100352

56. Sigler A (2010) Use of colchicine to prevent recurrence of ear keloids. A new approach. J Plast Reconstr Aesthet Surg 63:e650 652. https://doi.org/10.1016/j.bjps.2010.03.018

57. Daga D, Singh RK, Pal US, Gurung T, Gangwar S (2017) Efficacy of oral colchicine with intralesional hyaluronidase or triamcinolone acetonide in the Grade II oral submucous fibrosis. Natl J Maxillofac Surg 8:50-54. https://doi.org/10.4103/njms. NJMS_5_17

58. Ozdemir O, Calisaneller T, Sonmez E, Kiyici H, Caner H, Altinors N (2010) Topical use of colchicine to prevent spinal epidural fibrosis in rats. Neurol Res 32:1117-1120. https://doi. org/10.1179/016164110x12681290831487

59. Muntoni S, Rojkind M, Muntoni S (2010) Colchicine reduces procollagen III and increases pseudocholinesterase in chronic liver disease. World J Gastroenterol 16:2889-2894. https://doi. org/10.3748/wjg.v16.i23.2889

60. Leung J, Bonis PA, Kaplan MM (2011) Colchicine or methotrexate, with ursodiol, are effective after 20 years in a subset of patients with primary biliary cirrhosis. Clin Gastroenterol Hepatol 9:776-780. https://doi.org/10.1016/j.cgh.2011.05.010

61. Nikolaidis N, Polyzos SA, Giouleme O et al (2016) Colchicine to decrease inflammation and fibrosis in patients with metabolic dysregulation. Med Hypotheses 95:34. https://doi.org/10.1016/j. mehy.2016.08.006

62. Shah S, Das S, Jain A, Misra DP, Negi VS (2020) A systematic review of the prophylactic role of chloroquine and hydroxychloroquine in coronavirus disease-19 (COVID-19). Int J Rheum Dis. https://doi.org/10.1111/1756-185x.13842

63. Ahn BY, Kang CK, Seo JD et al (2020) A case of breakthrough COVID-19 during hydroxychloroquine maintenance. J Korean Med Sci 35:e231. https://doi.org/10.3346/jkms.2020.35.e231

64. Sattui SE, Liew JW, Graef ER et al (2020) Swinging the pendulum: lessons learned from public discourse concerning hydroxychloroquine and COVID-19. Expert Rev Clin Immunol. https:// doi.org/10.1080/1744666x.2020.1792778

65. Gurjar M, Agarwal V (2020) Usefulness of hydroxychloroquine for COVID-19: does answer lie in timing to start? Indian J Rheumatol (epub ahead of print)

66. Akpovwa H (2016) Chloroquine could be used for the treatment of filoviral infections and other viral infections that emerge or emerged from viruses requiring an acidic $\mathrm{pH}$ for infectivity. Cell Biochem Funct 34:191-196. https://doi.org/10.1002/cbf.3182

67. Kumar A, Liang B, Aarthy M et al (2018) Hydroxychloroquine inhibits zika virus NS2B-NS3 protease. ACS Omega 3:1813218141. https://doi.org/10.1021/acsomega.8b01002

68. Parra-Medina R, Sarmiento-Monroy JC, Rojas-Villarraga A, Garavito E, Montealegre-Gómez G, Gómez-López A (2020) 
Colchicine as a possible therapeutic option in COVID-19 infection. Clin Rheumatol 39:2485-2486. https://doi.org/10.1007/ s10067-020-05247-5

69. Scarsi M, Piantoni S, Colombo E et al (2020) Association between treatment with colchicine and improved survival in a single-centre cohort of adult hospitalised patients with COVID-19 pneumonia and acute respiratory distress syndrome. Ann Rheum Dis. https ://doi.org/10.1136/annrheumdis-2020-217712

70. Haslak F, Yildiz M, Adrovic A et al (2020) Management of childhood-onset autoinflammatory diseases during the COVID-19 pandemic. Rheumatol Int 40:1423-1431. https://doi.org/10.1007/ s00296-020-04645-x

71. Heneka MT, Golenbock D, Latz E, Morgan D, Brown R (2020) Immediate and long-term consequences of COVID-19 infections for the development of neurological disease. Alzheimers Res Ther 12:69. https://doi.org/10.1186/s13195-020-00640-3

72. Salehi S, Reddy S, Gholamrezanezhad A (2020) Long-term pulmonary consequences of coronavirus disease 2019 (COVID19): what we know and what to expect. J Thorac Imaging 35:W87-W89

73. Ahmed S, Zimba O, Gasparyan AY (2020) Thrombosis in coronavirus disease 2019 (COVID-19) through the prism of Virchow's triad. Clin Rheumatol 39:2529-2543. https://doi.org/10.1007/ s10067-020-05275-1

74. Grabias B, Kumar S (2016) Adverse neuropsychiatric effects of antimalarial drugs. Expert Opin Drug Saf 15:903-910. https://doi. org/10.1080/14740338.2016.1175428

75. Eo DR, Lee MG, Ham DI et al (2017) Frequency and clinical characteristics of hydroxychloroquine retinopathy in Korean patients with rheumatologic diseases. J Korean Med Sci 32:522-527. https ://doi.org/10.3346/jkms.2017.32.3.522

76. Marmor MF, Kellner U, Lai TY, Melles RB, Mieler WF (2016) Recommendations on screening for chloroquine and hydroxychloroquine retinopathy (2016 revision). Ophthalmology 123:13861394. https://doi.org/10.1016/j.ophtha.2016.01.058

77. Fiehn C, Ness T, Weseloh C, et al. (2020) Safety management in treatment with antimalarials in rheumatology. Interdisciplinary recommendations on the basis of a systematic literature review. Z Rheumatol. https://doi.org/10.1007/s00393-020-00785-4(epub ahead of print)

78. Mason JW (2017) Antimicrobials and QT prolongation. J Antimicrob Chemother 72:1272-1274. https://doi.org/10.1093/jac/ dkw591

79. Patil A, Jerang Y, Mathew J (2020) Hydroxychloroquine-induced auditory toxicity. Indian J Rheumatol 15:53-55

80. Bogdanos DP, Daniil Z, Zakynthinos E, Gourgoulianis K, Sakkas LI (2020) When there is a pandemic there is no time to waste: should we have hydroxychloroquine in our armoury against COVID-19 infected patients? Mediterr J Rheumatol 31:94-97. https://doi.org/10.31138/mjr.31.1.94

81. Paliani U, Cardona A (2020) COVID-19 and hydroxychloroquine: is the wonder drug failing? Eur J Intern Med. https://doi. org/10.1016/j.ejim.2020.06.002
82. Howard SC, McCormick J, Pui CH, Buddington RK, Harvey RD (2016) Preventing and managing toxicities of high-dose methotrexate. Oncologist 21:1471-1482. https://doi.org/10.1634/theon cologist.2015-0164

83. Lee JS, Oh JS, Kim YG, Lee CK, Yoo B, Hong S (2020) Methotrexate-related toxicity in patients with rheumatoid arthritis and renal dysfunction. Rheumatol Int 40:765-770. https://doi. org/10.1007/s00296-020-04547-y

84. Ajmani S, Preet Singh Y, Prasad S et al (2017) Methotrexateinduced pancytopenia: a case series of 46 patients. Int J Rheum Dis 20:846-851. https://doi.org/10.1111/1756-185x.13004

85. Lee W-I, Hissaria P (2019) Drug-induced interstitial lung disease. Indian J Rheumatol 14:19-26. https://doi.org/10.4103/09733698.272161

86. Slobodnick A, Shah B, Krasnokutsky S, Pillinger MH (2018) Update on colchicine, 2017. Rheumatology (Oxford) 57:i4-i11. https://doi.org/10.1093/rheumatology/kex453

87. Haj Yahia S, Ben Zvi I, Livneh A (2018) Colchicine intoxication in familial Mediterranean fever patients using clarithromycin for the treatment of Helicobacter pylori: a series of six patients. Rheumatol Int 38:141-147. https://doi.org/10.1007/s00296-017-3823-1

88. Tal R, Semo Oz R, Amarilyo G et al (2020) Safety and efficacy of intravenous colchicine in children with familial Mediterranean fever. Rheumatol Int 40:121-128. https://doi.org/10.1007/s0029 6-019-04348-y

89. Grossman C, Farberov I, Feld O, Livneh A, Ben-Zvi I (2019) Efficacy and safety of long-term treatment with intravenous colchicine for familial Mediterranean fever (FMF) refractory to oral colchicine. Rheumatol Int 39:517-523. https://doi.org/10.1007/ s00296-018-04237-w

90. Finkelstein Y, Aks SE, Hutson JR et al (2010) Colchicine poisoning: the dark side of an ancient drug. Clin Toxicol (Phila) 48:407-414. https://doi.org/10.3109/15563650.2010.495348

91. Gasparyan AY, Ayvazyan L, Cocco G, Kitas GD (2012) Adverse cardiovascular effects of antirheumatic drugs: implications for clinical practice and research. Curr Pharm Des 18:1543-1555. https://doi.org/10.2174/138161212799504759

92. Folegatti PM, Ewer KJ, Aley PK et al (2020) Safety and immunogenicity of the ChAdOx1 nCoV-19 vaccine against SARSCoV-2: a preliminary report of a phase $1 / 2$, single-blind, randomised controlled trial. Lancet. https://doi.org/10.1016/S0140 $-6736(20) 31604-4$.

Publisher's Note Springer Nature remains neutral with regard to jurisdictional claims in published maps and institutional affiliations. 